\title{
PSYCHOLOGISCHE ASPEKTE DER ALTERSBEDINGTEN KINESOPHOBIE
}

Die Erscheinung* eines alten Menschen verbindet sich in unserer Vorstellung mit einem dazugehörenden Requisit, nämlich einem Ruhesessel für Pensionäre. Manche Menschen glauben, daß die sog. körperliche Beweglichkeit ein Markmal der Kindheit, bzw. der Reifejahre sei. Der Verzicht auf körperliche Beweglichkeit im vorge - schrittenen Alter sei - nach bestimmter Meinung - - etwas völlig Normales, ja Notwendiges.

Was sagt uns die Psychologie darüber?

Wir möchten diesen Vortrag auf folgende Weise gliedern:

1) Erklärung der Terminologie

2) Einige Untersuchungen über Kinesophobie

3) Physiologische Folgen der Kinesophobie

4) Psychische Folgen der Kinesophobie

5) Psychologischer Hintergrund der Furcht vor eigener Beweglichkeit

6) Grundzüge einer psychologischen Kinesotherapie

7) Abschlußbemerkung

\section{ERKLÄRUNG DER TERMINOLOGIE}

Für die hier anwesenden Zuhörer, die vielleicht mit der psychologischen oder psychiatrischen lexikalischen Terminologie nicht im einzelnen vertraut sind, wollen wir bestimmte termini erklären.

Die zusammengesetzten Wörter, in denen der Termin ,kinè" vorkomment, entstammen dem griechischen Wort: to kínema, kinematos, d.h. Bewegung; kinetikós (kinetos): z. B. kinetós anèr, ist adjektivisch und bedeutet: bewegliche...; infinitiv; kinèin, bedeutet: sich bewegen; Phobie - (gr.) phobia - bedeutet neuropathologische Furcht vor etwas; phobos - (gr.) - Furcht. In unserem Vortrag wird von einer Furcht vor Bewegung gesprochen, also von einer Kinesophobie. Wenn jemand aber

* Poniższy referat został wygloszony na Uniwersytecie Jana Guttenberga w Moguncji $29 \times 1984$ r. 
sich gern und viel bewegt, so sprechen wir von einer Kinesophilie (gr.): philèin - lieb haben, lieben, für etwas Herz haben, gern haben usw.

Menschen, die an neuropathologischer Furcht vor eigener Bewegung leiden, regeneriert man durch verschiedene Bewegungsarten. Diese Behandlung nennen wir Kinesotherapie. Hier würde es wohl nützlich sein noch auf den Unterschied zwischen „Angst” und „Furcht” bzw. „Phobie” hinzuweisen. Die Psychologie sieht folgenden Bedeutungsunterschied bei diesen Wörtern: „Angst” heißt ein mit Beengung, Erregung, Verzweiflung verknüpftes Lebensgefühl, dessen besonderes Kennzeichen die Aufhebung der willensmäßigen und verstandesmäßigen „Steuerung” der Persönlichkeit ist. Man sieht auch in der Angst einen aus dem Gefahrenschutzinstinkt erwachsenen Affekt, der teils in akutem Ausbruch (dem Schreck verwandt), teils in schleichendquälender Form eine elementare Erschütterung bewirkt. In der Psychoanalyse stellt Angst einen Zentralbegriff dar und ist nach Freud im eigentlichen Sinne Trennungsangst (z.B. Angst des Säuglings bei der Trennung von der Mutterbrust, die Kastrationsangst in der Ödipussituation usw.) ${ }^{1}$.

„Fuchrt” wird als Lebensgefühl des Bedrohtseins verstanden. In mancher Beziehung ist Furcht der Angst verwandt, sie hält gewöhnlich länger an. Wenn Angst beim unmittelbaren Erscheinen einer unerwarteten, drohenden Person oder eines ekelerregendes Objektes entsteht, so ist Furcht immer mit der in der Vorstel - lungskraft (Phantasie) vergegenwärtigten Situation oder einem vergegenwärtigten Objekt vergeknüpft. Es handelt sich dann auch immer um etwas Unerwünschtes oder dem Individuum oft Feindliches.

„Phobie” ist eine zwanghaft auftretende Furcht vor bestimten äußeren - manchmal auch inneren - Objekten oder Situationen, ohne daß eine wirkliche Gefahr besteht. Die Phobie - auch die hier beschriebene Kinesophobie - ist mehr Furcht als Angst im obenangegebenen Sinne. Die Grenzen zwischen diesen psychischen Zuständen sind allerdings oft schwer zu ziehen.

EINIGE UNTERSUCHUNGEN VON ERSCHEINUNGSFORMEN DER KINESOPHOBIE

Sowohl die ältere als auch die moderne Medizin, die Gerontologie, Geriatrie und die Rehabilitationspsychologie beweisen, daß die körperliche Beweglichkeit von einer prinzipiellen Bedeutung für die allgemeine Gesundheit des Menschen ist. Auch in meiner neuesten Untersuchung von Aktivitäten bei den älteren Priestern zeigte sich, daß ein Teil von

1 Vgl. F. Dorsch, Psychologisches Wörterbuch, Hamburg-Bern 1963, S. 17. 
ihnen von der Kinesophobie betroffen war (vgl. Henryk Piszkalski, Proces adaptacji do przejścia na emeryturę u starszych kapłanów, Kraków 1983, 250; übersetzt: Adaptationsprozeß beim Übergang in den Ruhestand bei den älteren Priestern. Eine psychologische Studie).

Was die körperliche Beweglichkeit anbelangt, so gab es unter den Befragten zwei verschiedene Gruppen. Manche von ihnen lebten gern isoliert und vermieden Kontakte mit anderen Menschen, sie blieben körperlich wenig aktiv, beteten, schrieben etwas, ließen sich eher besuchen, als daß sie anderen einen Besuch machten. Sie saßen den ganzen Tag in ihren Wohnzimmern. Dabei dachten sie viel über ihre eigenen Probleme und ihre Gesundheit nach, im Gespräch wandten sie sich der ,schönen, harmonischen" Vergangenheit zu und bedauerten, wie wenig sie jetzt am ,Übel” der Welt etwas zu ändern vermögen. Manchmal waren sie einsilbig (unkommunikativ), in eigene offenbar pessimistische Gedanken versunken.

Die Kontaktscheu, eine Paralellerscheinung der Kinesophobie, führte immer zu einem schwachen sog. „Informationsmetabolismus”, wie sich der berühmte polnische Psychiater Dr. A. Kępiński ausdrückt, also zu einem geringen und inhaltsarmen Informationsaustausch und $\mathrm{zu}$ einer schwachen Informations - Verarbeitungs kapazität der älteren Person ${ }^{2}$. Die anderen - in der Mehrheit - zeigten sich aufgeschlossen und aktiv, gingen viel spazieren, unternahmen kleine oder auch längere Reisen, verrichteten erfreuliche Hobbyarbeiten, pflegten auch im Haushalt beim Zimmerputzen zu helfen usw.

Im Unterschied zu den Vertretern der ersten Gruppe waren diese von einer heiteren Gesinnung. Sie hatten mehr Zeit fürs Gebet. In der Bewegungspraktik verbanden sie das Nützliche mit dem Angenehmen. Sie hielten sich an das Prinzip: „Fahren, wo man schon muß, gehen, aber wo immer man kann" Dies ist die Hauptregel der Kinesophilie.

Ich kann also auf Grund eigener Untersuchungen, wie auch nach eigenem freien Gespräch mit den Befragten, auf eine positive Einwirkung des Sich - Bewegens auf die körperliche und seelische Gesundheit schließen ${ }^{3}$. Schon an dieser Stelle drängt sich die Frage auf: Ist die Gesundheit Ursache oder Folge des Sich - Bewegens?

Viel spricht für die zweite Annahme. Besonders scheint sich das zu beweisen im hohen Alter, wenn man als Pensionär oder als Rentner Herr der eigenen Zeit ist. Im hohen Alter aber hängt die Beweglichkeit mehr von dem Subjekt selbst ab, als von den objektiven Verhältnissen, zudem kann sich der Mensch diese selber gestalten.

2 A. K ępi ński, Schizofrenia, Warszawa 1974, S. 45.

${ }^{3} \mathrm{H}$. Piszkalski, a. a. O., S. 163. 
Betrachten wir zunächst die physiologischen Erscheinungen im menschlichen Organismus als Folge einer altersbedingten Unbeweglichkeit (Kinesophobie). Die Einschränkung der Mobilität durch eine sitzende Lebensweise im Übermaß, z.B. durch sitzende oder liegende Position Stunden hindurch oder unbewegliches Stehen (Lift), verursacht, allgemeingesagt, ein schlechteres Funktionieren des gesamten Organismus wie auch seiner einzelnen Teile.

Infolge der Unbeweglichkeit sinkt die Oxydation von Lunge, He:z und Kreislauf. Auch die Oxydationsfähigkeit von Muskeln und Gehirnrinde läßt nach. Infolge der Immobilität arbeiten Nieren, Leber und Magen schlechter. Behindert ist auch der Stoffwechsel und sein Metabolismus. Das Blut erreicht nur schwach extremere Körperteile, z.B. die Zehen u.ä. Mangel an körperlicher Bewegung wirkt sich besonders negativ aus an Organen des sog. bradytrophischen Zellengewebes, in dem der Wechsel von Nährsubstanzen durch Diffusion erfolgt (Durchdringen, Überschwemmen). und nicht durch Kapillargefäße, wie es im tachytrophischen Zellengewebe der Fall ist. Zum bradytrophischen Zellengewebe gehören: Augenlinse, Hornhaut, Zähne, Knorpel, Knochen, Sehnen und bestimmte Teile der Gefäßwände - die innere Membrane und teilweise die mittelere Schicht ${ }^{4}$. In diesem Zellengewebe könnem am ehesten Degenerationserscheinungen auftreten, besonders als Ablagerungen in den Gelenkeknorpeln, in der Augenlinse und an den Wänden der Arterien. Bewegungslosigkeit oder Verharren in nur einer Körperstellung (Sitzen, Knieen, Stehen, Liegen) wirkt negativ auf die Elastizität der Glieder, verursacht ihr Steifwerden, ihre Leistungsminderung oder verursacht sogar körperlichen Schmerz.

Es versteht sich, daß bei geminderter Funktionsfähigkeit des menschlichen Organismus alles in ihm langsamer abläuft - und im bradytrophischem Gewebe wird der Erneuerungsprozess noch zusätzlich verzögert - er ist dazu weniger hygienisch, weniger gesundheitsfördernd, weniger ergiebig. Es läßt sich somit behaupten, daß der weniger durchblutete, wenig ,bewegte" Organismus auch weniger widerstandsfähig ist gegenüber verschiedenen Indispositionen und Krankheiten. Viele Abwehrkräfte des Organismus bleiben infolge lange anhaltender Bewegungslosigkeit unaktiviert, weshalb dann der gesamte Organismus leichter schädlichen Faktoren in der Umgebung der betreffenden Person unterliegt.

${ }^{4}$ M. Bürger, Biomorfoza $i$ jej znaczenie $w$ procesie starzenia się $i$ stanach chorobowych, Warszawa 1965, S. 4-5. 
Die Scheu vor Beweglichkeit im vorgerückten Alter, also altersbedingte Kinesophobie, zieht nicht nur negative physiologische Folgen nach sich, sondern hat auch zehlreiche unerwünschte Wirkungen im Bereiche des menschlichen Seelen - lebens.

Die verlangsmate bzw. erschwerte Aktivität der inneren Vorgänge verlangsamte im Organismus trübt auch das Allgemeinbefinden des Menschen. Dieser Zustand beeinflußt seinerseits die gesamten Reaktionen des Denkens, des Gefühls und des Willens. Verursacht durch eine unzureichende Durchblutung des Gehirns, tritt eine verringerte Perzeption bei Denkprozessen ein. Das Interesse an neun Denkinhalten wird schwächer. Es senkt sich das Niveau des sog. Informationsmetabolismus. Der emotionale Bereich erfährt eine Verarmung infolge der Verengung des Personenkreises und des Umfangs von Vorgängen, die nur durch tätiges (bewegliches) Hinwenden erreichbar sind.

Der zu keiner Anstrengung herausgeforderte Wille, z.B. bei Bewältigung einer gewissen Trägheit der Bewegung, wird schwach und verliert nach einiger Zeit die frühere Spontaneität, da er nicht mehr die Möglichkeit hat, die ihm eigene Dynamik zu entwickeln.

Mit einem Wort gesagt, infolge einer anhaltenden Kinesophobie vermindert sich die geistige Aufnahmefähigkeit des Menschen. Dies zieht eine Verarmung an neuen Erfahrungen im geistigen und emotionalen Bereich nach sich. Die Abnahme von Erlebnissen nach Quantität und Qualität erzeugt einen Mangel an Anteil - nahme an dem, was „,neu”, ,,interessant”, ,informativ" ist. Es entsteht somit die Situation eines „,irculus vitiosus" von lauter negativen Bedingtheiten: die verringerte Beweglichkeits- Aktivität vermindert die geistige Aufnahmefähigkeit, und diese hinwiederum veranlaßt das Individuum, sich der Unbeweglichkeit hinzugeben. Derartige Erscheinungen können sich häufen, stärker werden und schließlich sich in einen Dauer - zustand verwandeln.

Welches sind die am meisten ins Auge fallenden Merkmale dieses psychischen Zustandes?

Bei einem Individuum, das sich der Verführung zur Kinesophobie nicht entgegenzustellen vermag, läßt sich ein beständiges Mißtrauen in die eigenen Möglichkeiten beobachten, wenn es um die eigene physische Kraft sowie um das in ihr vorhandene latente und tatsächliche Vermögen geht. Im letzten Ergebnis glaubt das Individuum an das eigene ,Unvermögen" und nimmt nun für immer eine Haltung ein, die ihr die nicht bewältigte Furcht vorschreibt - eine Phobie vor eventueller Übermüdung. So entsteht darüber hinaus ein negatives Bild (Autoporträt) 
seiner selbst als eines schwachen, gebrechlichen, zur gröseren Anstrengung unfähigen Menschen, der beständiges Ausruhen benötigt, der von anderen gemieden wird, der andere behindert und der fast unnütz ist.

\section{PSYCHOLOGISCHER HINTERGRUND DER FURCHT VOR EIGENER BEWEGLICHKEIT}

Es drängt sich die Frage auf: warum meidet eine Person im vorgerückten Alter die beschriebene Beweglichkeit, warum empfindet sie sogar eine gewisse Abscheu vor ihr? Es scheint, daß die Schwierigkeiten des eigenen Organismus nur zum Teil diese Einstellung erklären, denn gewisse Abnutzungserscheinungen im Atmungsbereich, im Muskel-Gelenk-System und im Blutkreislauf genügen nicht zur Begründung dieser im Alter häufigen Kinesophobie. Der Grund für die Immobilität einiger Rentner liegt eher im psychischen als im organischen Bereich.

Folgende psychische Haltungen kommen in Frage:

1. Unbegründete Furcht vor möglicher Erschöpfung.

2. Haltloser Widerwille gegenüber einer körperlichen Anstrengung.

3. Einbildung, unfähig zu sein zur Überwindung einer normalen Schwierigkeit beim spürbaren Widerstand im Organismus.

4. Unberechtigte Meinung, jedes Greisenalter müsse gebrechlich sein.

5. Nur die Bequemlichkeit suchen, obwohl sie die vorzeitige "Taperigkeit" des Denkens begünstigt.

6. Schematisches Denken und ein gewisser, jeden wohlwollenden Rat von Fachleuten ablehnender Alterstarrsinn.

7. Falsch verstandener sog. Lebensernst und Furcht vor Lächerlichkeit bei praktizierter „Beweglichkeit”.

Wie ersichtlich, sind dies Elemente der Psychophobie und nicht Behinderungen seitens des gealterten Organismus des Menschen. Die oben erwähnten Quellen der Kinesophobie, wie sie im vorgerückten Alter auftreten, sind zahlreich und erst jetzt genau bestimmt. Sie erschöpfen nicht alle möglichen Variationen. Sie können in Erscheinung treten nach überstandenen Krankheiten, durch frühere Beeinflussung durch Umwelt und Erziehung. Sie können verursacht sein auch durch Übermaß an Fürsorge von Personen, die einen besonderen Einfluß auf das gegebene Individuum haben. Es kann auch eine Art von Hypochondrie vorliegen, die sich in einer krankhaften beständigen Unruhe und Sorge um die eigene Gesundheit und das eigene Leben zeigt. 
Angesichts des Problems der Kinesophobie muß man von der Überlegung ausgehen, daß der Mensch - trotz der angetroffenen Schwierigkeiten - imstande ist, sie zu beherschen, ja sogar eine begrenzte $\mathrm{Be}-$ weglichkeit liebzugewinnen, wie z.B. das in der USA verbreitete jogg ing, eine praktizierte „Beweglichkeit”. Der Anblick älterer grauhaariger Personen im Sportdreß beim Lauf auf Bürgersteig oder rechten Straßenseite ist keine Seltenheit. Niemand geniert sich hier, niemand wundert sich. Die Menschen, die Bewegung lieben und also Freunde der Beweglichkeit (Kinesophilie) sind, werden als Personen angesehen, die in richtiger Weise bemüht sind um ihren Körper wie auch um psychische Hygiene.

Die beständige und vor allem systematische Aufrechterhaltung der physischen Leistungfähigkeit und die damit verbundene günstige Beeinflussung des psychischen Wohlbefindens ist die billigste Arznei und der zugänglichste Weg bei einem Heilungsprozeß. Man nennt dies Kinesotherapie, d.h. also Heilung durch eine ruhige, gleichmäßige körperliche Bewegung.

Man soll sich allerdings immer vor einer Utberanstrengung in allen Bewegungsarten hüten. Vor allen Dingen muß man Acht darauf geben, damit das Sichbewegen immer ruhig, allmählich steigernd, niemals aber wild und hastig von dem Älteren ausgeübt wird, z.B. nicht plötzlich aufspringen, der Straßenbahn oder Taxi um jeden Preis nachlaufen u.ä.

Der Autor des US-Bestsellers über Joggen, der „Jogging-Papst” der 52 jährige Amerikaner $\operatorname{Jim~Fixx}^{6}$ ist beim Joggen in einem Wald in der Nähe von Hardwick, US-Bundesstaat Vermont, tot zusammengebrochen. Fixx ist laut Gerichtsmediziner einem $\mathrm{He}-$ rzinfarkt erlegen. Darüber wurde bekannt-gegeben in „Die Presse" - 23.7.84. Nach Ansicht von Odolf Gruber, einem zwölfmaligem österreichischen Meisters im Marathonlauf, habe Jim Fixx ganz einfach zuwenig Kondition gehabt und sei zu ,schwächlich” gewesen. Sein Buch hatte 1978 Milionen von Amerikanern, ,auf die Beine" gebracht und wurde in zwölf Sprachen übersetzt. Der ehemalige Journalist hatte sich nach einem Unfall im Jahre 1967 ,,gesundgelaufen", mehr als 30 Kilogramm abgenommen (zuvor hatte er 100 Kilogramm gewogen und nach eigenen Angaben täglich zwei Päckchen Zigaretten geraucht). Ab dann lief Fixx täglich 15 Kilometer. „Laufen ist überhaupt der sesündeste Sport, den es gibt. Damit werden Herz und Kreislauf gestärkt, man nimmt ab und kommt an die frische Luft" - versichert der 64 jährige Leiter von „Jogging-Center” in Wien-Leopoldstadt, Adolf Gruber. „Von

${ }^{6}$ J. Fix x, The Complete Book of Running, New York 1977. 
angenommen 1000 Personen, die joggen, kann für 20 bis 30 Menschen diese Tätigkeit lebensrettend sein" - erklärt Dozent Dr. Paul Haber Wien (,Die Presse - 24.7.84). Natürlich, man muß ,nichts übertreiben” (Adolf Gruber).

Aber nun, nochmals zurück zu unserer einfachen, allgemein zugänglichen Beweglichkeit. Bei der „Anwendung” dieses Heilmittels bedarf. es gewöhnicher Impulse des Willens. Es geht darum, sich zu zwingen, von jeder Möglichkeit Gebrauch zu machen, etwa nach dem bekannten Grundsatz: „Fahren, wenn nötig, gehen wo immer möglich”. Es scheint, daß Anomalien, wie sie in der Beschleunigung des Alterns und in schnell voranschreitenden sklerotischen Erscheinungen zum Ausdruck kommen, ihre Wurzeln haben im intellektuellen, emotionalen und voluntarischen Bereich der beschriebenen Personen, aber auch in der Tätigkeit des Gehirns.

Der Ganzheit des hier dargestellten Problems halber wollen wir noch eine Art von Beweglichkeit erwähnen: die Geistesarbeit. Auch die bringt den menschlichen Organismus in eine gewisse „Bewegung”. Man versteht das auf folgende Weise: Die psychisch-seelische Aktivität (das Denken) wird jeweils von einem komplizierten, kettenartigen, biochemischen Stoffwechsel begleitet, der durch gleichzeitige Blutbereicherung eine bessere Oxydation der Gehirnzellengewebe ermöglicht. Die Geistesarbeit ist also auch gesund für den alternden Körper, und gleichzeitig verzögert sie auch die Arterienverkalkung.

Intellektuelle Einsicht, Bewältigung emotionaler Komplexe und vor allem eine Anspannung des Willens machen sich bezahlt. Die Kinesotherapie muß zu einer Kinesophilie führen. Die Psychologie ist bemüht, den Patienten bei diesem Prozeß zu helfen, d.h. also zu heilen durch Gespräch, Überzeugung, Ermutigung, wobei sie auf eventuelle medizinische Mithilfe rechnet.

Wie schon oben ausgeführt, kann man den Eindruck haben, daß krankhafte Erscheinungen, die sich z.B. in der Beschleunigung des Alterns, besonders auch bei der Arterienverkalkung äussern, auf psychische Einflüsse zurückzuführen sind. Damit sind die drei seelischen Schichten der Persönlichkeit gemeint: die intellektuelle, die emotionale und die woluntarische. In Bezug auf die Überwindung der Kinesophobie im hohen Alter hat das letzte Wort nicht der Organismus, sondern der Wille. Die Kinesotherapie führt über die Aktivierung des Willens zur Überwindung von irrationalen Gefühlskomplexen. Wenn das gelungen ist, wird aus der Kinesophobie die wünschenswerte $\mathrm{K}$ in esophilie. 